\title{
El recalce con micropilotes para la conservación de un muro de tierra compactada realizado con la técnica del tapial
}

\section{The technique of underpinning with micropiles in conserving a compacted earth wall using "tapial-technic"}

$\underline{\text { I. Valverde-Palacios }}^{(*)}$, R. Fuentes ${ }^{(*)}$, I. Valverde-Espinosa ${ }^{(*)}$, M. Martín-Morales ${ }^{(*)}$, J. Santos-Sánchez $^{(*)}$

\section{RESUMEN}

Durante el proceso de ejecución de un aparcamiento público subterráneo en la ciudad de Granada (España) se descubrió una obra de ingeniería hidráulica periurbana de $55 \mathrm{~m}$ de longitud con tres tajamares (declarado BIC en el 2007) ligado a la margen izquierda del río Genil. Se detectaron tres fases constructivas, muro monolítico (s. XI), tapia real (s. XII-XIII) y una reparación posterior (s. XIX) a base de cajones de mampostería con machones e hiladas de ladrillo. Para la conservación de este elemento en su posición original se ejecutaron: micropilotes verticales en su perímetro; otros horizontales en su base; viga riostra de encepado y bulones con barra Gewi pasantes a la viga de arriostramiento. Durante la excavación, se ejecutó un entramado de perfiles metálicos laminados «L» soldados entre sí y a los micropilotes. El elemento está totalmente integrado en la obra subterránea y en el entorno del Paseo del Violón.

Palabras clave: Patrimonio Arquitectónico; conservación; muro de tierra; micropilotes.

\section{ABSTRACT}

In this paper, the discovery of a peri-urban work of hydraulic engineering is presented. A wall-dike was found during the excavation for an underground public parking on the left bank of the Genil River in Granada city (Spain). Those consist in a compacted earth wall (using "Tapial-technic") of $55 \mathrm{~m}$ length with three cutwaters. Three different construction methodologies were detected in the wall-dike: monolithic-wall (s. XI), royal wall (s. XII-XIII) and contemporary reparation (s.XIX). These elements were conserved in the original position with the help of the micropiles technique. The consolidation stages were as follows: vertical micropiles in its perimeter; horizontals micropiles in the base of the wall; brace beam and thought bolts with Gewi rod. After the digging, also, laminated metal sections with an "L" were welded together and to vertical micropiles. That element is fully integrated with both underground construction and its environment, "Paseo del Violón".

Keywords: Architectural heritage; conservation; compacted earth wall; micropiles.

(*) Universidad de Granada (España)

Persona de contacto/Corresponding author: nachoval@ugr.es (I. Valverde-Palacios)

Cómo citar este artículo/Citation: Valverde-Palacios, I., Fuentes, R., Valverde-Espinosa, I., Martín-Morales, M., Santos-Sánchez, J. (2014). El recalce con micropilotes para la conservación de un muro de tierra compactada realizado con la técnica del tapial. Informes de la Construcción, 66(534): e023, doi: http://dx.doi.org/10.3989/ic.12.131.

Licencia/License: Salvo indicación contraria, todos los contenidos de la edición electrónica de Informes de la Construcción se distribuyen bajo una licencia de uso y distribución Creative Commons Reconocimiento no Comercial 3.o. España (cc-by-nc). 


\section{INTRODUCCIÓN}

En muchos casos, la construcción de nuevas obras, tanto civiles como de edificación, comportan hallazgos casuales de ejemplos de la arquitectura antigua, hecho que es muy común en la ciudad de Granada. En este caso se presenta el sistema constructivo utilizado para la conservación de una obra de ingeniería hidráulica periurbana consistente en un muro o dique de tierra compactada, realizado con la técnica del Tapial. Éste fue descubierto durante el proceso de ejecución de un aparcamiento público subterráneo en el casco urbano de la ciudad de Granada (España). Dicho descubrimiento fortuito obligó a la paralización temporal de las obras y, debido al carácter histórico de los restos, a la ejecución de una actividad arqueológica de urgencia realizada por Gespad-AlAndalus.S.L. (1).

Concretamente, la zona de intervención se localiza en la margen izquierda del río Genil, próxima a la confluencia de uno de sus afluentes, el río Darro, en el denominado Paseo del Violón (Figura 1). En este sector, fuera del Centro Histórico, existen numerosos elementos periurbanos (2) (3), tales como la almunia Alcázar Genil y una rábita o morabito musulmán, hoy llamada ermita de San Sebastián, ambos construidos en el 1218.

Actualmente, las murallas que rodeaban toda la ciudad de Granada histórica han quedado relegadas a los lienzos que existen en las tres colinas que describe Bosque Maurel (3): la del Cerro de San Miguel, la de Alcazaba Cadima y la de la Alhambra. Estos elementos esenciales constituyen un testimonio vivo de su pasado histórico; su color rojizo y trazado, adaptado al terreno, hace que se pierdan en este emplazamiento topográfico tan característico del entorno de la ciudad en el sector NE.

Las investigaciones llevadas a cabo permiten diferenciar tres tipologías de muro de tierra y cal compactada, todas realizadas con la técnica del tapial, empleadas en la construcción de los lienzos de murallas granadinas: hormigón de cal o muro monolítico, tapia real y tapia calicastrada (5) (6) (7).

En la evolución del método constructivo de las distintas murallas, las diferencias más significativas corresponden al volumen relativo de tierra amasada con cal, su aporte y a la disposición de los diferentes niveles que la contienen, así como a los procedimientos constructivos que dan origen a los revestimientos. En esta evolución constructiva (del s. XI al XIV), se pasa de un muro monolítico (hormigón de cal) a tierra simplemente compactada protegida por un revestimiento grueso de mortero de cal (tapia calicastrada). En épocas intermedias, se intercalaban capas de tierra y cal en togadas más o menos separadas (tapia real).

El muro de tierra encontrado en la margen izquierda del río Genil constituía un dique de protección para evitar las inundaciones de las alamedas, jardines, huertas y almunias situadas en la llanura de inundación, aguas abajo del puente de igual nombre, donde se produce un aumento considerable de caudal por la confluencia de los ríos Genil y Darro. Además de este muro de tierra y cal compactada, que llegaba hasta el Puente Genil, se descubrió un muro contemporáneo constituido por cajones de mampostería con machones e hiladas de ladrillo y tres tajamares que se atribuye a mejoras y reparaciones posteriores. Pareja (8), en los años 70, describe el descubrimiento de otro gran lienzo de muro que discurría paralelo al cauce del río Genil a unos $2 \mathrm{~m}$ bajo la rasante de aquella época, frente al actual colegio P.P. Escolapios, aguas arriba del citado puente (Figura 1).

En este artículo se presenta el sistema constructivo empleado para la conservación del dique en su posición original mientras se llevaban a cabo los trabajos de excavación del solar hasta la cota requerida (del orden de $9 \mathrm{~m}$ ), manteniendo suspendido el muro a la cota en la que se localizó. Además, se estudió la coexistencia del muro con el parking público para conseguir la mejor integración con la menor pérdida de superficie posible.

\section{INTERPRETACIÓN HISTÓRICA DEL MURO O DIQUE DE CONTENCIÓN}

El río Darro y Genil han sido el escenario de numerosas crecidas y desbordamientos, sobre todo del primero cuando aún no estaba controlada la cuenca por la presa de Canales. La crecida documentada más antigua del Darro, en época nazarí, tuvo lugar tras una tormenta de gran intensidad el 24 de Abril de 1478. Ésta causó importantes inundaciones en el casco histórico de la ciudad de Granada (sectores del Zacatín, la Alcaicería,parte de la Mezquita Mayor y plaza de Bib-Rambla). Ayala-Carcedo et al. (9) describen más de veinte inundaciones asociadas al río Darro en el período que va desde 1478 a 1951.

No existen documentos escritos anteriores al s. XV acerca de estas avenidas, no obstante, el hallazgo de este dique o muro de contención hace pensar que estos acontecimientos, en el sector de la confluencia con el río Genil, se han sucedido desde tiempos inmemoriales. La presencia, en esta obra de ingeniería hidráulica, de varias tipologías constructivas distintas demuestra que esta zona ha sido siempre motivo de una atención especial. Dichas tipologías se pueden ordenar en el tiempo teniendo en cuenta su similitud constructiva con las cercas de los antiguos núcleos urbanos de las diferentes épocas de la Granada árabe. Si bien, datar con precisión el muro descubierto resulta complicado pues existe una gran diferencia respecto a los materiales utilizados: Formación $\mathrm{Al}$ hambra en las murallas y el aluvial del río Genil en el muro de su margen izquierda, constituido por tierra y cal dispuestas en obra mediante compactación. La utilización de distintos materiales provoca distorsiones, al menos visuales, aunque se hayan utilizado el mismo sistema constructivo (Técnica del Tapial). Además, de origen más reciente, se reconoce una tercera tipología con estructura de ladrillo. En conjunto, los restos encontrados presentan una longitud de $55 \mathrm{~m}$, un ancho variable entre 1,20 y $3 \mathrm{~m}$ y una altura máxima de $4 \mathrm{~m}$ (Figura 2).

A continuación se describen las tres tipologías constructivas reconocidas en el dique descubierto:

- Muro monolítico (hormigón de cal). Está constituido por tierra y áridos de distintos tamaños (de arena a cantos) aglomerados con cal $(\sim 10 \%)$ puestos en obra mediante compactación manual utilizando la técnica del Tapial. Presenta un aspecto compacto y un buen estado de conservación, sobre todo en su trasdós, ya que éste no ha sufrido la erosión provocada por el embate del agua. No obstante, la cara exterior no está tan deteriorada como era de esperar por lo que cabe pensar en reconstrucciones a lo largo del 


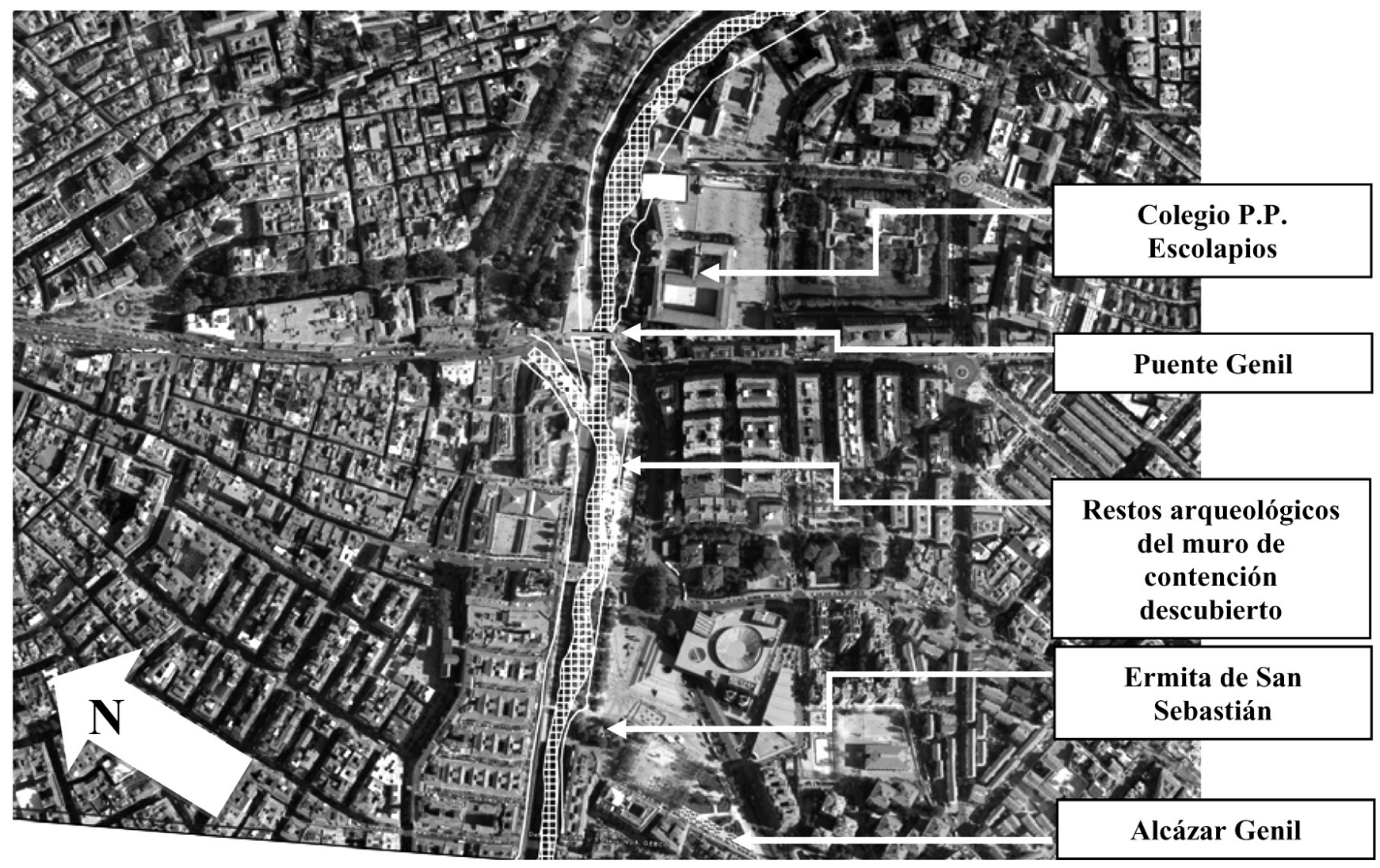

Figura 1. Situación de los restos del dique o muro de contención descubiertos en la excavación. La localización del muro de contención encontrado en la margen izquierda del ríos Genil coincide con el trazado antiguo del cauce (trama y líneas blancas finas) obtenido del grabado de R. Contreras de 1872 (4).

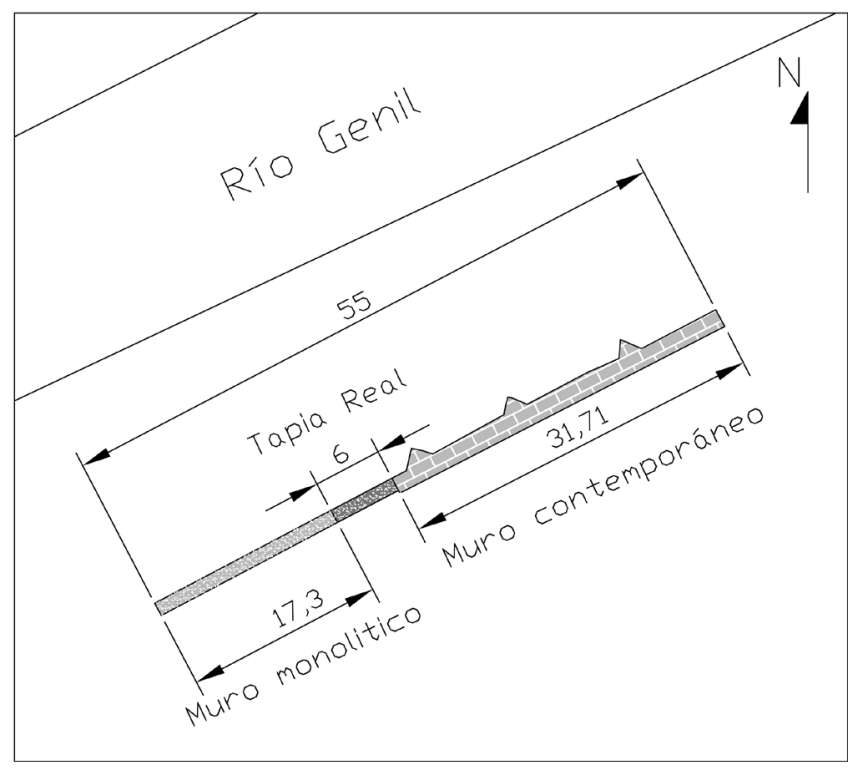

Figura 2. Representación esquemática en planta del muro descubierto con las distintas tipologías constructivas (cotas en metros).

tiempo mediante la aplicación de enfoscados con morteros de cal. Actualmente, presenta una longitud de $\sim 17 \mathrm{~m}$, con una discontinuidad longitudinal que tiende a marcar un recrecimiento posterior (Figura 3). Teniendo en cuenta su tipología constructiva, su situación periurbana y su similitud con las murallas de Granada del s. XI, puede establecerse que fue construido, al menos la cimentación y el tramo inferior, por esta época.

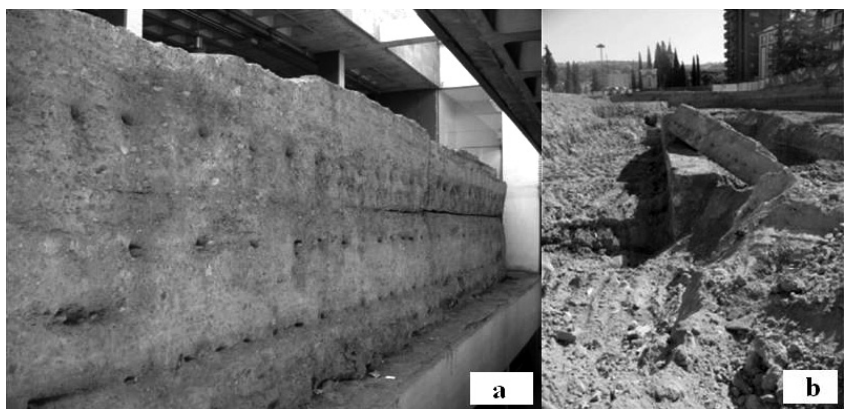

Figura 3. a) Muro monolítico en su estado actual, en el que se aprecia la discontinuidad longitudinal; b) vista del mismo muro en el momento de su descubrimiento, donde éste se rompió por la discontinuidad. Posteriormente, el tramo desplomado se colocó en su posición original.

- Tapia Real. Está constituida por la alternancia de capas de tierra y tierra con cal, de uno a dos centímetros de espesor, compactadas manualmente (Figura 4). En este caso, el revestimiento resulta directamente del propio método constructivo apareciendo de forma continua en todo el lienzo al producirse afluencia hacia los bordes del hormigón, mortero o lechada de cal durante el proceso de compactación. Aparece a continuación del anterior en dirección hacia el Puente Genil con un desarrollo de unos $6 \mathrm{~m}$ hasta alcanzar la estructura de uno de los tres tajamares. Siguiendo el criterio de datación utilizado en el caso del muro monolítico, este muro se construyó entre los siglos XII-XIII. $\mathrm{Su}$ existencia parece responder a reparaciones que fueron necesarias por la destrucción del muro monolítico en el sector más crítico, es decir, donde se producía la unión de 


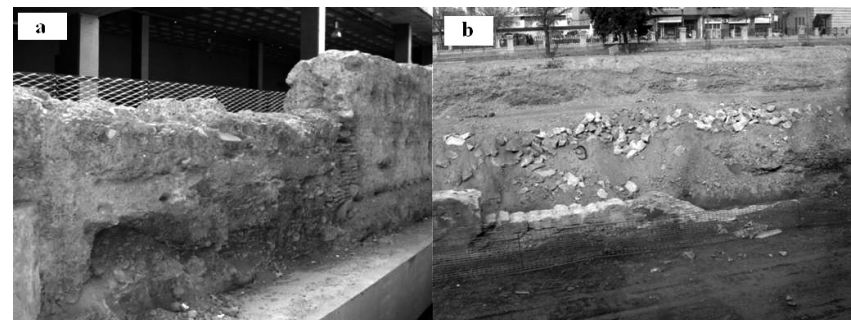

Figura 4. Vista actual de la tapia real (a), y cabecera de ésta en el momento del hallazgo (b).

las aguas del Genil y Darro. Prueba de ello es que en el s. XIX fue necesaria otra reparación que da lugar a la tercera tipología.

- Muro contemporáneo. Esta tipología consiste en cajones de mampostería conformados por machones e hiladas de ladrillo. En varios puntos de su base, conectando con la cimentación del muro se dispusieron tres tajamares para disminuir y contrarrestar la embestida de la corriente de agua contra el muro (Figura 5). Según el archivo municipal del Ayuntamiento de Granada, en 1886 se comunicó «que se había caído en el Paseo del Violón un trozo de paredón desde la ermita de San Sebastián al Puente Genil». Posteriormente, fue reparado mediante este sistema constructivo, por lo que este muro se construyó a finales del s. XIX.

El muro y dique de contención con tajamares del cauce del río Genil fue declarado Bien de Interés Cultural (BIC), en la categoría de monumento, mediante Decreto 193 de 26 de junio de 2007 (10). Además, tanto el Palacio Alcázar Genil como la Ermita de San Sebastián fueron declarados «Monumento Histórico Artístico» en 1922 y «Monumento Histórico Nacional» en 1931, respectivamente, catalogados igualmente como BIC.
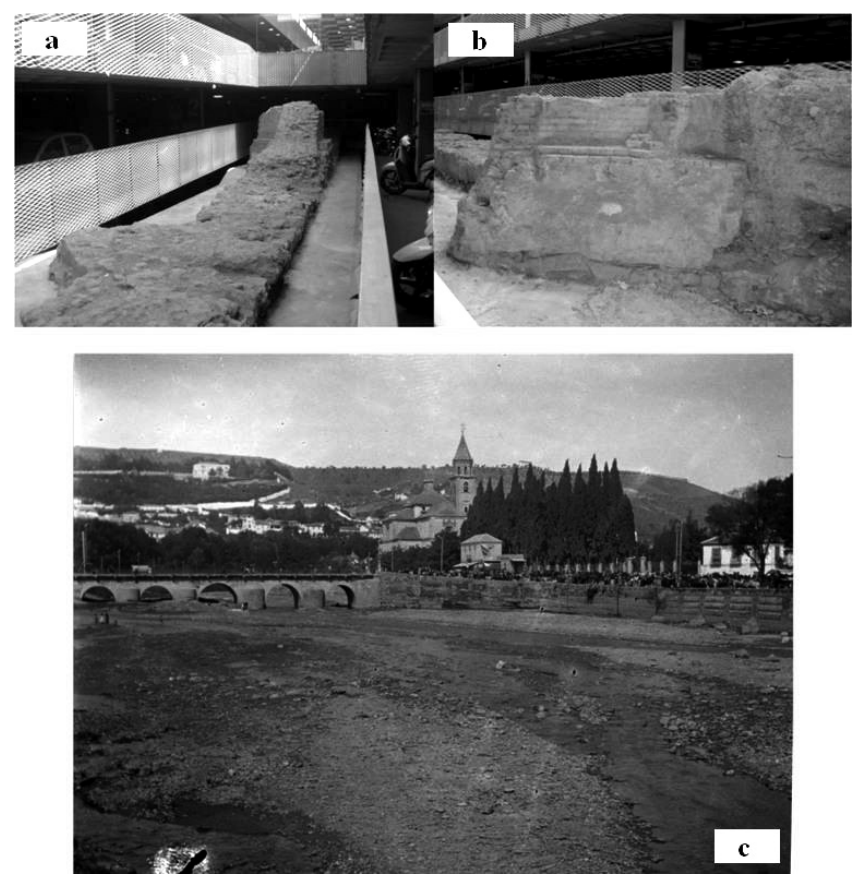

Figura 5. a) Muro contemporáneo. Se pueden observar las hiladas de ladrillo; b) Detalle de uno de los tajamares; c) fotografía tomada en el año 1907 (Colección fotográfica del Archivo del Ayuntamiento de Granada, España. Donada por J. Algarra en 1990) donde se observa al fondo a la derecha los cajones de mampostería y los tres tajamares.

\section{TÉCNICA EMPLEADA PARA LA CONSERVACIÓN}

Para la conservación del dique de contención en su posición original se empleó la técnica del recalce con micropilotes verticales y horizontales encepados con una viga de hormigón armado. Además, en dicha viga se dispusieron unos bulones horizontales y en los micropilotes verticales se soldaron unos perfiles laminados en forma de L.

Este proceso constructivo, llevado a cabo por la empresa SITE S.A., hizo posible mantener todo los tramos del muro (monolítico, tapia real y de ladrillo) en equilibrio a la vez que se llevaba a cabo el proceso de vaciado para alojar el parking (del orden de $9 \mathrm{~m}$ ).

A continuación se describe con detalle cada una de las fases del proceso constructivo para la conservación del dique en su posición original (Figura 6).

- Fase 1. Realización de micropilotes con una armadura tubular 114-9 mm en todo el perímetro de la muralla, con un diámetro de perforación de $180 \mathrm{~mm}$ y $14 \mathrm{~m}$ de longitud. Se dispusieron con una separación de 2 m y un empotramiento de $5 \mathrm{~m}$ en el aluvial de río Genil que está constituido por arenas y gravas, material que se reconoce desde la cota de apoyo de la base del muro. Los cálculos que se efectuaron establecen las siguientes resistencias estructurales del micropilote: a compresión 98,11 T, a tracción 122,8 T, a flexión 4,52 T m y a cortante 49,66 T. En el cálculo geotécnico de micropilotes inyectados, partiendo del empotramiento y tipo de terreno indicado, se establece una resistencia a tracción de 26,38 T aplicando el método de Bustamante (11) (12) con un coeficiente de seguridad de 2.

- Fase 2. Ejecución de perforaciones horizontales debajo del muro equipadas con armadura tubular 140-10 mm., e inyectadas al igual que los micropilotes con lechada de cemento. Esta medida complementaria contribuye a rigidizar la estructura y evitar el punzonamiento del muro, actuando como una «cama» para soportar el peso del mismo por la base.

- Fase 3. Arriostrado de las cabezas de todos los micropilotes con una viga de atado de hormigón armado formado un rectángulo. Dicha viga hace que los micropilotes actúen conjuntamente y transmitan las cargas del peso del muro al suelo aluvial a través de la fricción de éste con el tramo de fuste empotrado. Además, dota al conjunto de la rigidez suficiente para que el muro no se punzone. El dique se protegió con porexpan para evitar el contacto entre éste y el hormigón de la viga de atado, impidiendo así la modificación del color, porosidad y textura del muro, además de la transmisión de posibles cargas diferenciales.

- Fase 4. Realización de bulones con barra Gewi, de 50 mm de diámetro, pasantes a la viga de arriostramiento, con una longitud de $5 \mathrm{~m}$ y separación entre ejes de $3 \mathrm{~m}$, que aseguran y refuerzan el conjunto. Una vez fijado uno de sus extremos a la viga perimetral se tensaron por el lado opuesto a $5 \mathrm{~T}$, transmitiendo una compresión horizontal en la cimentación del muro.

- Fase 5. Excavación debajo del muro y posterior ejecución de entramado de perfiles metálicos laminados en «L» de 7 ó $10 \mathrm{~cm}$ de ala. Dichos perfiles se unieron, mediante soldaduras longitudinales y transversales a los micropilotes formando una celosía. Este sistema constructivo confería la rigidez y estabilidad necesaria para evitar el pandeo de 


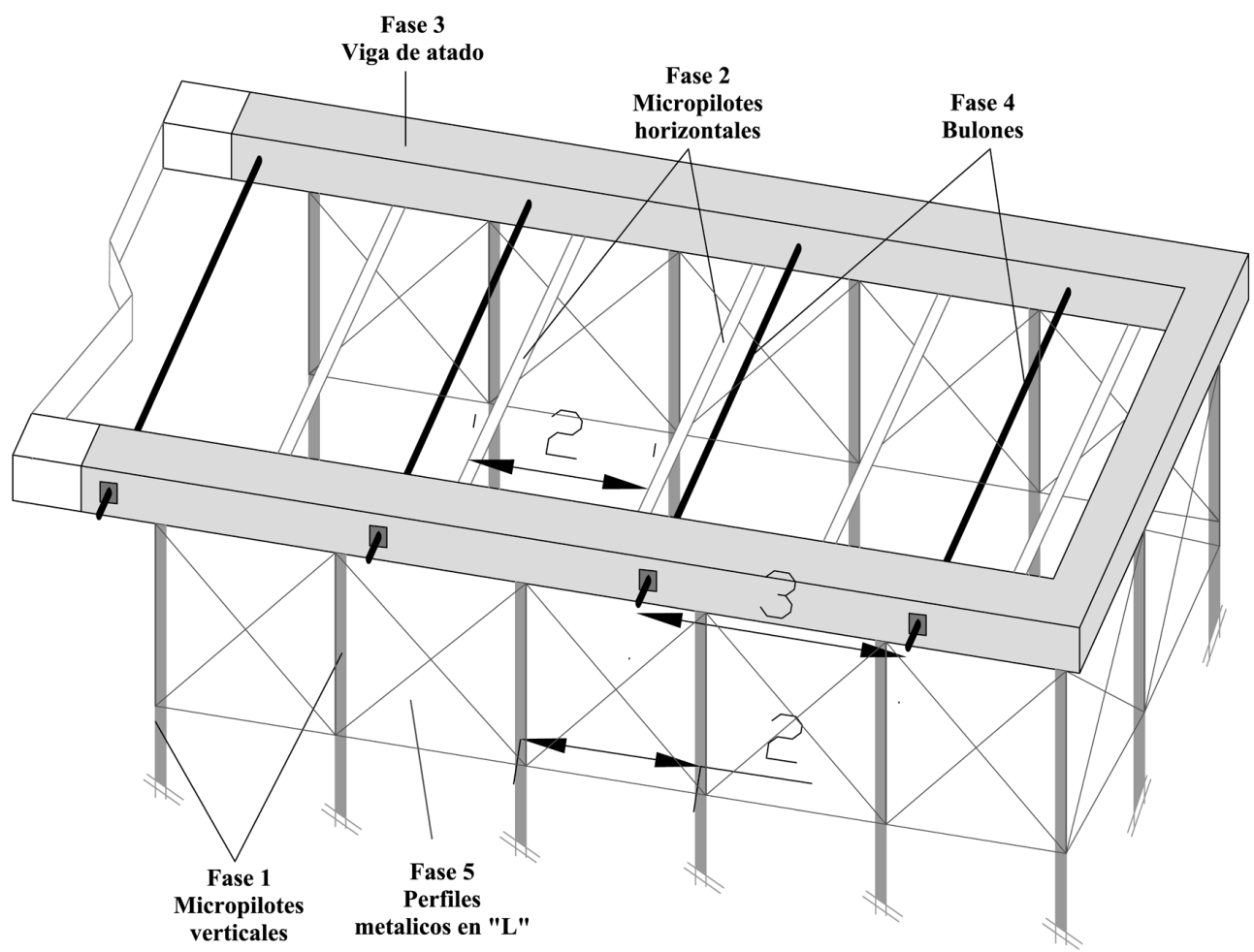

Figura 6. Dibujo esquemático de las fases constructivas realizadas para la conservación del muro en su posición original.

los micropilotes verticales que soportaban todo el peso del muro (Figura 7).

- Fase 6. Una vez que se alcanzó la cota máxima de excavación, del orden de $9 \mathrm{~m}$, se ejecutó la losa de cimentación del parking y conforme se realizaban los forjados se iban cortando los micropilotes hasta llegar al nivel -1 de sótano donde actualmente está ubicado el muro (Figura 8).

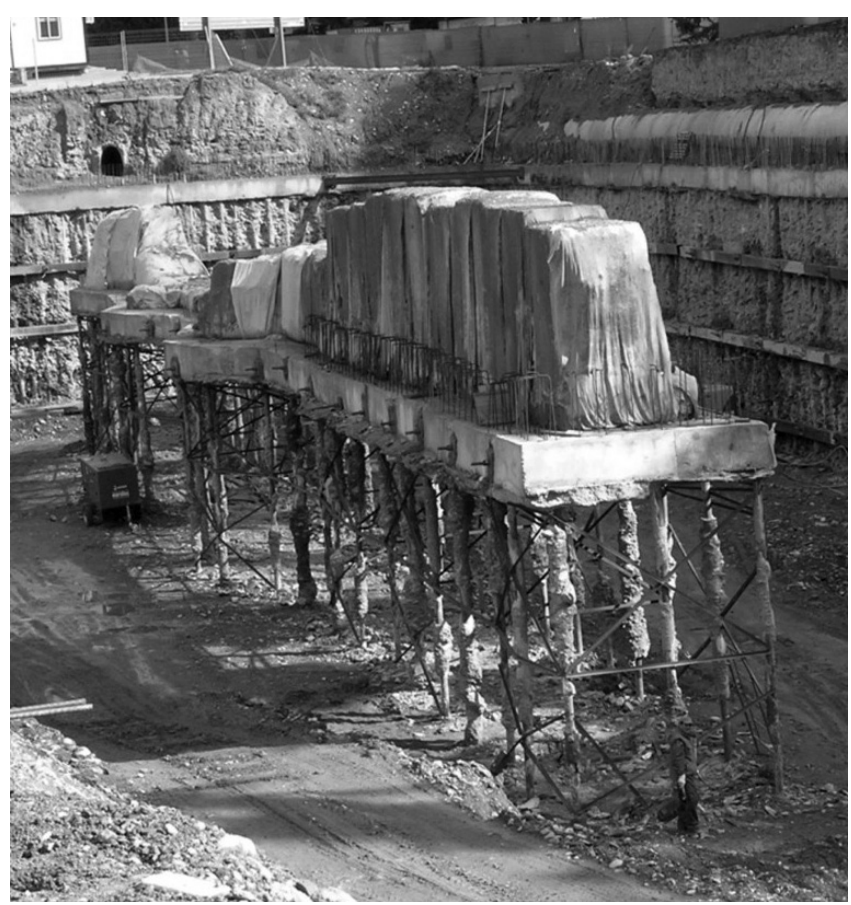

Figura 7. Fase final para la conservación del muro en su posición original. Se pueden apreciar los micropilotes, bulones y perfiles laminados en L.
- Fase 7. Ejecución de pilares apantallados de diferentes alturas, tres de ellos con ménsula de canto variable. Posteriormente, en la base se dispuso una losa de hormigón armado que engloba a la viga de encepado perimetral (Figura 9).

\section{CONCLUSIONES}

El método y sistema constructivo, así como la ejecución, han resultado muy adecuados para la conservación del dique con tajamares en su emplazamiento original, es decir, en la margen izquierda del río Genil en las proximidades de la confluencia de éste con el río Darro.

Es de destacar la importancia del sistema para la conservación de este y otros tipos de muros ya que ha sido válido para actuar en un desarrollo lineal de $55 \mathrm{~m}$ con anchuras variables

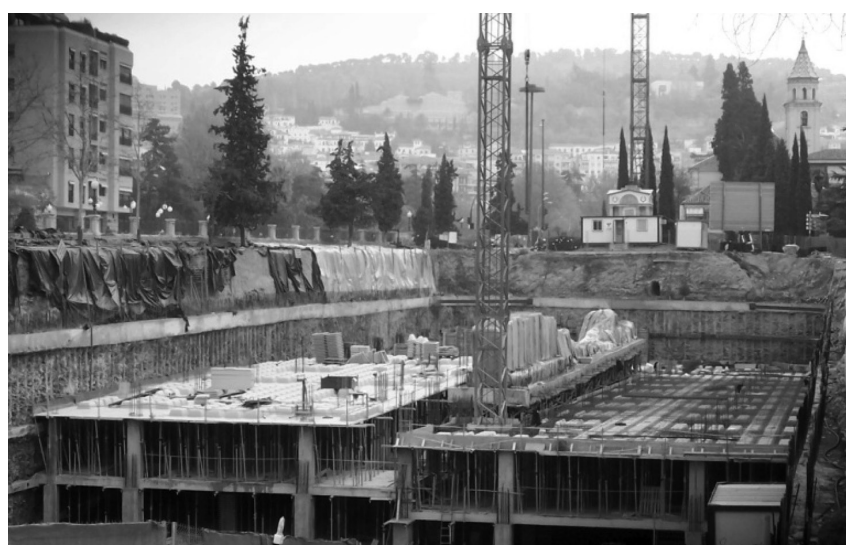

Figura 8. Muro inmerso en el proceso constructivo del parking (Fotografía cedida por SITE S.A.) 


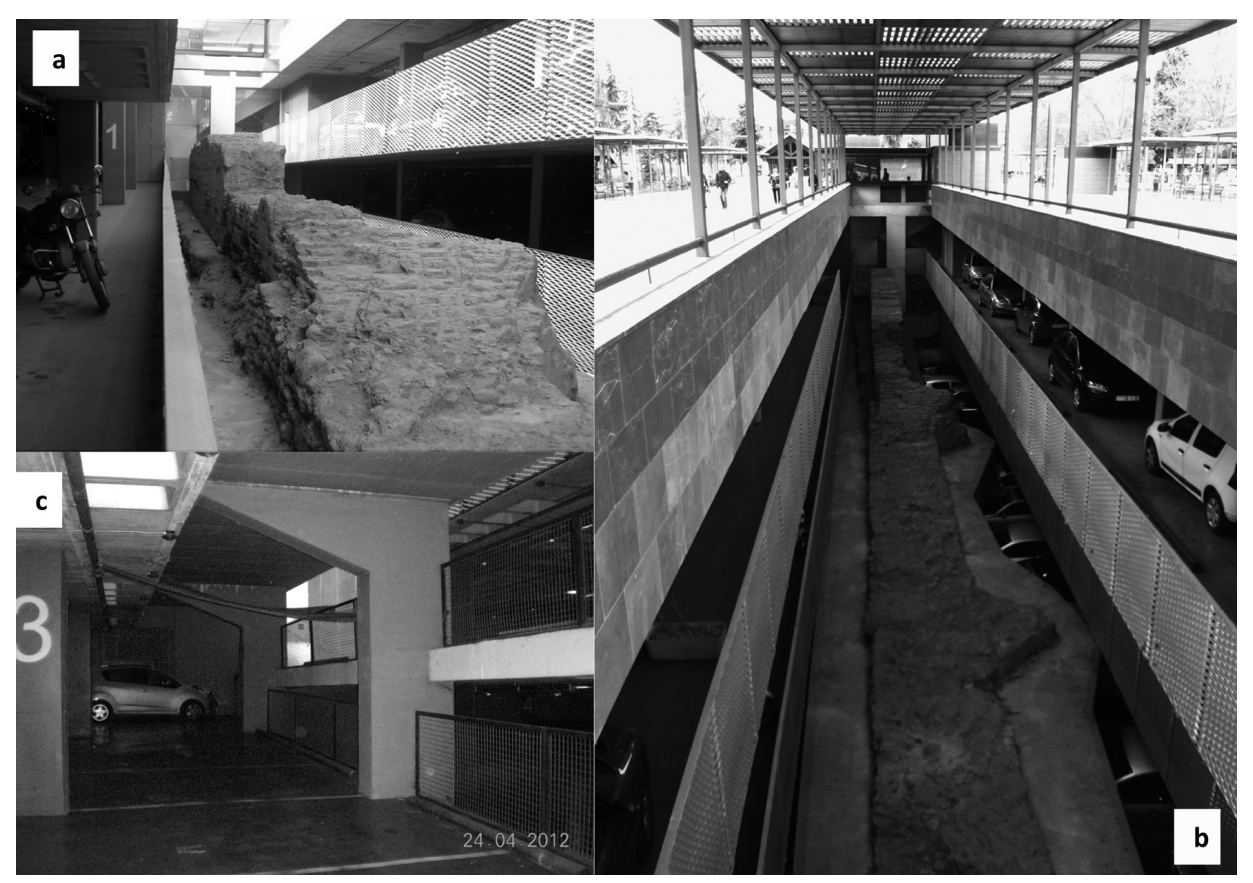

Figura 9. a) Vista del muro desde el nivel -1; b)Vista actual del dique a través de un hueco realizado en el último forjado del parking; c) Pilares apantallados con ménsula de canto variable en el nivel -3 que sustenta la losa sobre la que descansa el muro.

entre 0,85 y 3 m y una altura máxima de $4 \mathrm{~m}$, perdiendo la mínima superficie útil en el parking que se limita a las dimensiones de planta indicadas en el nivel -1 .

El elemento está totalmente integrado en la obra subterránea y en el entorno del Paseo del Violón dejando visible este BIC tanto dentro del parking como desde la plaza que se ha desa- rrollado tras la construcción del parking a través de un hueco en el último forjado.

\section{AGRADECIMIENTOS}

Queremos agradecer la colaboración de las empresas Gespad-Al-Andalus.S.L. y SITE S.A.

\section{REFERENCIAS}

(1) García Consuegra-Flores, J., Rodríguez-Aguilera, A. (2005). Actividad arqueológica de urgencia en el Paseo del Violón $s / n$, Granada, Informe Técnico. Granada: GESPAD Al-Andalus S.L.

(2) Torres-Balbás, L. (1950). Los contornos de las ciudades hispano-musulmanas en Al-Andalus, XV.pp. $477-478$.

(3) Bosque-Maurel, J. (1988). Geografía urbana de Granada (Ed. Facs.). p. 68. Granada: Universidad de Granada.

(4) Contreras-Muñoz, R. (1872). Plano de Granada con el recinto de sus antiguas murallas y monumentos árabes, guía de calles y plazas y dependencias oficiales e indicador de los edificios más notables históricos o artísticos. Recursos de investigación de la Alhambra. http://hdl.handle.net/10514/633.

(5) Valverde-Espinosa, I., Gallego-Roca, F. J., López-Osorio, J. M. (1992). Arquitectura de tierra en el Albaicín de Granada. En Congreso Internacional: rehabilitación del patrimonio arquitectónico y edificación, (pp. 204-209). Tenerife: CICOP.

(6) Ontiveros-Ortega, E. (1995). Estudio de los materiales empleados en la construcción de los tapiales de las murallas de Granada (Tesis Doctoral), pp. 277-278. Granada: Universidad de Granada.

(7) Algorri-García, E., Vázquez-Espí, M. (1996). Enmienda a dos de los errores más comunes sobre el tapial. En de las Casas, A., Huerta, S., Rabasa, E. (Eds.) Actas del Primer Congreso Nacional de Historia de la Construcción, (pp. 19-23). Madrid: I. Juan de Herrera, CEHOPU.

(8) Pareja-López, E. (1982). Granada. p. 387, Granada: Inst. Provincial de Estudios y Promoción Cultural.

(9) Ayala-Carcedo, F.J., Rodríguez-Ortiz, J.M., Prieto-Alcolea, C., Durán-Valsero, J.J., Lamas-Romero, J.L., Rubio-Amo, J. (1986). Mapa previsor de riesgos por inundaciones en núcleos urbanos de Andalucía y Extremadura. p. 64, Madrid: IGME.

(10) Junta de Andalucía. (2007). Decreto 193/2007, de 26 de junio. Boletín Oficial de la Junta de Andalucía, (132): $24-26$.

(11) Bustamante, M. (1980). Capacité d'ancrage et comportament des tirants injectes scelles dans une argile plastique (Tesis doctoral). París: Ecole Nationale de Ponts et Chaussees.

(12) Bustamante, M. (1986). Un método para el cálculo de los anclajes y de los micropilotes inyectados. Boletín de Información del Laboratorio de Carreteras (CEDEX), (174): 3-23. 